\title{
Effect of an aqueous Russian tarragon extract on glucose tolerance in response to an oral dextrose load in non-diabetic men
}

This article was published in the following Dove Press journal:

Nutrition and Dietary Supplements

2 March 20II

Number of times this article has been viewed

\author{
Richard J Bloomer' \\ Robert E Canale' \\ Ivo Pischel ${ }^{2}$ \\ 'Cardiorespiratory/Metabolic \\ Laboratory, The University of \\ Memphis, Memphis, TN, USA; \\ 2PhytoLab GmbH \& Co. KG, \\ Vestenbergsgreuth, Germany
}

Correspondence: Richard J Bloomer Cardiorespiratory/Metabolic Laboratory, I6IF Roane Field House, The University of Memphis, Memphis, TN 38I52, USA Tel + | 90 I-678-434 |

Fax + | 90|-678-359|

Email rbloomer@memphis.edu
Background: Russian tarragon extracts have been reported to have anti-diabetic activity in animals. This pilot study aimed to investigate the acute effects of an aqueous extract of Russian tarragon (RT) on serum glucose and insulin in response to an oral glucose tolerance test (OGTT).

Methods: Using a randomized, double-blind, cross-over design, 12 non-diabetic men reported to the lab on 2 different mornings separated by 1 to 2 weeks, and ingested $75 \mathrm{~g}$ of dextrose in solution. Fifteen minutes before ingestion, subjects ingested either $2 \mathrm{~g}$ of RT or a placebo. Blood samples were collected before ingestion of the RT and placebo, and at 15 , $30,45,60$, and 75 minutes post ingestion of the dextrose load. Samples were assayed for serum glucose and insulin.

Results: For serum glucose, no condition $(P=0.19)$ or condition $\times$ time $(P=0.99)$ effect was noted. A time effect was noted $(P<0.0001)$, with values at 15 and 30 minutes higher than pre-ingestion $(P<0.05)$. No area under the curve (AUC) effect $(P=0.54)$ was noted, although a $4.5 \%$ reduction in AUC was observed for RT $\left(569 \pm 92 \mathrm{mg} \cdot \mathrm{dL}^{-1} \cdot 75 \mathrm{~min}^{-1}\right) \mathrm{vs}$ placebo $\left(596 \pm 123 \mathrm{mg} \cdot \mathrm{dL}^{-1} \cdot 75 \mathrm{~min}^{-1}\right)$. Similar findings were noted for serum insulin, with no condition $(P=0.24)$ or condition $\times$ time $(P=0.98)$ effect noted. A time effect was noted $(P<0.0001)$, with values at 15,30 , and 45 minutes higher than pre-ingestion $(P<0.05)$. No AUC effect $(P=0.53)$ was noted, although a $17.4 \%$ reduction in AUC was observed for RT $\left(114 \pm 22 \mu \mathrm{IU} \cdot \mathrm{mL}^{-1} \cdot 75 \mathrm{~min}^{-1}\right)$ vs placebo $\left(138 \pm 30 \mu \mathrm{IU} \cdot \mathrm{mL}^{-1} \cdot 75 \mathrm{~min}^{-1}\right)$. Approximately two-thirds of subjects ingesting the RT experienced attenuation in both the glucose and insulin response to the OGTT.

Conclusion: These data indicate that acute ingestion of RT results in a slight, non-statistically significant lowering of blood glucose in response to a dextrose load. This occurs in the presence of a slightly lower insulin response. These findings are specific to a small sample of healthy, non-diabetic men. Additional study is needed involving a larger sample of individuals with pre-diabetes or untreated diabetes.

Keywords: Artemisia dracunculus L., glucose, insulin resistance, nutritional supplements, Russian tarragon

\section{Background}

It is estimated that 24 million Americans are living with diabetes, while close to 60 million more have been diagnosed with pre-diabetes; the majority of which are type 2 in pathology. ${ }^{1}$ This escalating problem is not simply isolated to the United States, as the incident rate for this condition continues to rise throughout the developed world. ${ }^{2}$ For example, it has been reported that 285 million people worldwide have diabetes mellitus, with a projected growth of $6.5 \%$ per year, and 439 million affected 
by year $2030 .^{2}$ Indeed, a need exists to aid the millions of individuals with blood glucose regulatory problems.

Multiple methods are available to help control elevated blood glucose levels, including regular exercise, ${ }^{3}$ alteration in dietary intake, ${ }^{4}$ prescription drugs,${ }^{5}$ and nutritional supplements, ${ }^{6}$ but the latter has received a great deal of attention in recent years. In particular, herbal ingredients (often specialized extracts) have been studied for their potential glucose regulatory effects. ${ }^{7,8}$ While several candidate ingredients appear to have promise, including, eg, Trigonella foenum-graecum (fenugreek), Opuntia ficus-indica (prickly pear), Momordica charantia (bitter mellon), and Panax quinquefolius (American ginseng), of particular interest is Russian tarragon (Artemisia dracunculus L.), as it may manifest a genotype-specific insulin-sensitizing phenotype. ${ }^{9}$

Russian tarragon (RT) has been used for centuries in Russia and middle Asia as a digestive, diuretic, and antipyretic herbal medicinal agent. Today, RT is classified as a Generally Recognized as Safe (GRAS) material following the removal of the harmful components of the essential oil (eg, estragol and methyleugenol). Please see: (http://www. accessdata.fda.gov/scripts/fcn/fcnDetailNavigation. cfm?rpt=eafusListing\&id=3596 for tarragon extract (Artemisia dracunculus L.), Doc No. 2840; accessed January 6, 2011). An ethanol extract of RT has been developed and reported to exhibit anti-hyperglycemic activity, ${ }^{10}$ which may be enhanced when using a bioenhancer/solubilizer $\left(\right.$ Labrosol $\left.{ }^{\circledR}\right) .{ }^{11}$ From a mechanistic viewpoint, the clinical effects of RT on carbohydrate metabolism appear secondary to enhancing insulin signaling; specifically by decreasing the levels of PTP1B (a specific protein tyrosine phosphatase), while not significantly impacting IRS-1, IRS-2, PI-3 kinase, Akt, insulin receptor, or GLUT-4. ${ }^{12}$ The polyphenolic compounds 6-demethoxycapillarisin and 2',4'-dihydroxy-4methoxydihydrochalcone are believed to be chiefly responsible for the glucose-lowering activity of the RT. ${ }^{13}$

An aqueous extract of RT has also been developed and reported recently to provide similar effects on glucose lowering compared with an ethanol extract. ${ }^{14}$ In this study, an oral glucose tolerance test (OGTT) was administered to a group of rats following single oral ingestion of an aqueous or ethanol extract of RT (at a dosage of $6 \mathrm{mg} \cdot \mathrm{kg}^{-1}$ ). The diabetic drug glibenclamide was used for comparison (at a dosage of $18 \mathrm{mg} \cdot \mathrm{kg}^{-1}$ ). The glucose area under the curve (AUC) was similar but slightly lower for the aqueous extract than both the ethanol extract and glibenclamide. These data indicate that oral intake of an aqueous RT extract may provide benefits in terms of glucose regulation after an OGTT similar to glibenclamide, and possibly greater than an ethanol extract. However, to date, no published human trials are available on the use of RT to lower blood glucose in response to an OGTT. Therefore, the purpose of the present study was to investigate the effects of an aqueous RT extract on serum glucose and insulin in response to an OGTT.

\section{Methods \\ Subjects}

Twelve healthy, non-diabetic men were recruited from the University of Memphis campus and the Memphis, TN area. Subjects were not smokers, and had no diagnosed cardiovascular or metabolic disease. Subjects were not using dietary supplements known to influence blood glucose regulation. Subject characteristics are presented in Table 1. Health history, drug and dietary supplement usage, and physical activity questionnaires were completed by subjects. The study was approved by the university committee for human subject research. All subjects provided written informed consent.

\section{Laboratory visit I: screening}

During the initial visit to the laboratory, subjects completed the informed consent form, and health and physical activity questionnaires. Heart rate, blood pressure, height, weight, waist and hip circumference, and skinfold thickness (using a 7-site test with Lange calipers) were measured and used for subject description. Subjects were provided with food logs and instructions on how to complete these, the day before each test day (described below).

Table I Characteristics of the 12 men in the study

\begin{tabular}{lc}
\hline Variable & Data \\
\hline Age (years) & $24.8 \pm 5.3$ \\
Height $(\mathrm{cm})$ & $180.0 \pm 7.0$ \\
Weight $(\mathrm{kg})$ & $82.0 \pm 8.6$ \\
BMI $\left(\mathrm{kg} \cdot \mathrm{m}^{-2}\right)$ & $25.4 \pm 3.3$ \\
Body fat $(\%)$ & $13.1 \pm 5.0$ \\
Waist $(\mathrm{cm})$ & $83.5 \pm 7.8$ \\
Hip $(\mathrm{cm})$ & $102.4 \pm 6.1$ \\
Waist:hip & $0.82 \pm 0.05$ \\
Resting heart rate (bpm) & $55.5 \pm 7.3$ \\
Resting systolic blood pressure $(\mathrm{mmHg})$ & $116.5 \pm 11.0$ \\
Resting diastolic blood pressure $(\mathrm{mmHg})$ & $69.0 \pm 5.2$ \\
Years anaerobic exercise training & $6.3 \pm 6.9$ \\
Hours per week anaerobic exercise & $3.9 \pm 1.9$ \\
Years aerobic exercise training & $3.1 \pm 2.8$ \\
Hours per week aerobic exercise & $2.1 \pm 2.1$ \\
\hline
\end{tabular}

Notes: Data are mean \pm SD. 


\section{Laboratory visits 2 and 3: testing}

Subjects reported to the laboratory on two different days during the morning $(6: 00-8: 00 \mathrm{am})$ following a $\geq 10$ hour overnight fast. Subjects consumed in random order, separated by 1 to 2 weeks, either a placebo (cellulose) or an aqueous extract of RT (Finzelberg GmbH \& Co. KG, Germany). The extract of RT was prepared as follows: $1 \mathrm{~kg}$ of the raw material of Russian Tarragon herb (cultivated in Poland by Martin Bauer Polska Sp. z. o. o., Witaszyczki, Poland) was extracted twice with $6 \mathrm{~L}$ of water at $80^{\circ} \mathrm{C}$. After cooling, the water extracts were separated by filtration through paper filters and the solvent was evaporated by means of a rotatory evaporator. The dense extract obtained was mixed with $30 \%$ maltodextrin as a suitable carrier, and dried at $50^{\circ} \mathrm{C}$ in a vacuum drying chamber. The dried extract was adjusted for its particle size and filled in hard gel capsules. The analysis showed $0.2 \%$ to $0.7 \%$ total flavonoids (calculated using hyperoside as standard), with water content of less than $4 \%$ and a complete removal of the essential oil (Estragol, Methyleugenol). ${ }^{15}$ The placebo capsules were cellulose.

Subjects consumed the conditions in capsule form. Fifteen minutes after the ingestion of the RT or placebo, an OGTT was administered, which consisted of $75 \mathrm{~g}$ of $100 \%$ pure dextrose powder (NOW Foods, Bloomingdale, IL) mixed into $355 \mathrm{~mL}$ of water. Subjects consumed the entire solution within 2 minutes. The 15 minute time period was deemed appropriate based on prior unpublished experiments using RT, in particular when considering the duration of the post OGTT monitoring. Blood samples were taken before the ingestion of the RT or placebo (after a 10 minute rest period) and at 15, 30, 45, 60, and 75 minutes after ingestion of the $75 \mathrm{~g}$ dextrose load. While standard procedures for an OGTT include sampling every 30 minutes for 2 to 3 hours, ${ }^{16}$ we have noted that when using healthy, non-diabetic men as test subjects, such a time course is not optimal. Specifically, serum glucose values return to baseline values within 1 hour post ingestion. ${ }^{17}$ Therefore, extending the measurement period beyond this time is unnecessary. Hence, we opted for more frequent samples taken during the 75 minute post ingestion period.

Before each blood draw, heart rate (via 60 second palpation) and blood pressure (via auscultation) were taken. The rationale for these measures was based on the observation that a postprandial rise in serum glucose is associated with an increase in reactive oxygen species production, ${ }^{18,19}$ which is known to induce an acute state of endothelial dysfunction. ${ }^{19,20}$ This in turn may lead to vascular dysfunction and elevated blood pressure; however, we are unaware of any data linking the acute rise in reactive oxygen species to an elevation in heart rate. If the RT was effective at lowering the serum glucose response to the OGTT, it may also affect blood pressure. No food or calorie containing beverages were allowed during the entire test period, although water was allowed ad libitum and matched for each subject during both days of testing. These same procedures were followed for both test days.

\section{Blood processing and biochemistry}

Venous blood samples were taken from subjects via needle and Vacutainer ${ }^{\circledR}$ at all times indicated above. Blood samples were allowed to clot at room temperature and then were centrifuged at $1500 \times \mathrm{g}$ for 15 minutes at $4^{\circ} \mathrm{C}$. Samples were then stored at $-70^{\circ} \mathrm{C}$ until analyzed. Glucose was determined in serum using standard enzymatic (glucose oxidase) procedures as described by the reagent manufacturer (Thermo Fisher Scientific, Waltham, MA). Standard curves were developed for determination of unknown samples. Glucose assays were performed in triplicate. Insulin was determined in serum using a solid phase enzyme linked immunosorbent assay (ELISA) kit following the specific instructions of the manufacturer (Calbiotech, Spring Valley, CA). Insulin assays were performed in duplicate.

\section{Physical activity and dietary intake}

Subjects were asked to avoid strenuous physical activity during the 24 hours before each test day; as such activity may have influenced our results. ${ }^{21,22}$ Subjects were asked to record all food and drink consumed during the 24 hours prior to each test day. Subjects received a copy of this diet record and were asked to duplicate this intake during the 24 hours immediately before the second test day. All records were analyzed for total calories, protein, carbohydrate, fat, vitamin C, vitamin E, and vitamin A (Food Processor SQL, version 9.9, ESHA Research, Salem, OR).

\section{Statistical analysis}

Serum glucose and insulin data were analyzed using a 2 (condition) $\times 6$ (time) analysis of variance (ANOVA). Tukey post hoc testing was performed as needed. The area under the curve (AUC) was calculated for both glucose and insulin using the trapezoidal method as described in detail by Pruessner et al. ${ }^{23}$ Data are presented as mean \pm standard error of the mean. Subject descriptive characteristics are presented as mean \pm standard deviation. All analyses were 
performed using JMP statistical software (version 4.0.3, SAS Institute, Cary, NC). Statistical significance was set at $P \leq 0.05$.

\section{Results}

All 12 subjects successfully completed both test sessions. The RT and dextrose loads were well tolerated. No significant differences were noted between conditions for any measured dietary variable $(P>0.05)$. Table 2 presents dietary data for both the placebo and RT test days. For hemodynamic data, no condition $\times$ time or time effect was noted for any variable $(P>0.05)$. However, a condition effect was noted for all variables $(P \leq 0.05)$, each being lower for RT compared with placebo. Data are presented in Table 3.

\section{Biochemical measures}

For serum glucose, no condition $(P=0.19)$ or condition $\times$ time $(P=0.99)$ effect was noted. However, a time effect was noted $(P<0.0001)$, with values at the 15 and 30 minute collection times higher than pre-ingestion $(P<0.05)$. No AUC effect $(P=0.54)$ was noted, although a $4.5 \%$ reduction in glucose AUC was observed for RT compared with placebo. Of the 12 subjects, 7 responded to treatment, evidenced by a lower serum glucose response with RT compared with placebo. Data are presented in Figure 1.

For serum insulin, no condition $(P=0.24)$ or condition $\times$ time $(P=0.98)$ effect was noted. However, a time effect was noted $(P<0.0001)$, with values at the 15 , 30 , and 45 minute collection times higher than pre-ingestion $(P<0.05)$. No AUC effect $(P=0.53)$ was noted, although a $17.4 \%$ reduction in insulin AUC was observed for RT compared to placebo. Of the 12 subjects, 8 responded to treatment, evidenced by a lower serum insulin response with RT compared with placebo. Data are presented in Figure 1.

Table 2 Dietary data of 12 men during the 24 hours before ingestion of placebo and Russian tarragon

\begin{tabular}{lcc}
\hline Variable & Placebo & Russian tarragon \\
\hline Kilocalories & $2387 \pm 218$ & $2454 \pm 209$ \\
Protein $(\mathrm{g})$ & $153 \pm 22$ & $165 \pm 27$ \\
Carbohydrate $(\mathrm{g})$ & $288 \pm 42$ & $284 \pm 42$ \\
Fat $(\mathrm{g})$ & $73 \pm 10$ & $77 \pm 8$ \\
Vitamin C (mg) & $86 \pm 23$ & $70 \pm 16$ \\
Vitamin E (mg) & $7 \pm 2$ & $6 \pm 2$ \\
Vitamin A (RE) & $750 \pm 307$ & $720 \pm 236$ \\
\hline
\end{tabular}

Notes: Data are mean \pm SEM. No statistically significant differences were noted for kilocalories $(P=0.83)$, protein $(P=0.74)$, carbohydrate $(P=0.94)$, fat $(P=0.80)$, vitamin $C(P=0.58)$, vitamin $\mathrm{E}(P=0.63)$, or vitamin $\mathrm{A}(P=0.94)$.

\section{Discussion}

Our data do not provide statistically significant support for the ability of RT to alter glucose disposal favorably after an OGTT in healthy, non-diabetic men. However, roughly two-thirds of subjects ingesting the RT did experience attenuation in both the glucose and insulin response to the OGTT. Considering that all subjects were young, healthy, non-diabetic men, it is possible that further study with older individuals and/or those with impaired glucose tolerance would provide more robust effects. Moreover, as subjects simply ingested a single dosage of the RT 15 minutes before the OGTT, it is possible that chronic intake of the RT for a period of weeks leading up the OGTT (as for most oral hypoglycemic agents) may provide additional benefit. Further study is needed to investigate such hypotheses, possibly including both men and women.

In terms of overall effects, our data are not as strong as those obtained from the animal studies of Ribnicky et al ${ }^{11}$ and Walbroel et $\mathrm{al}^{14}$ who reported favorable effects on glucose tolerance in response to treatment with RT. It is possible that differences in the usual response between animals and humans contributed to these mixed findings, coupled with the fact that our subjects were all healthy, non-diabetic men. As stated above, while the condition effect for both glucose $(P=0.19$; Figure 1) and insulin $(P=0.24$; Figure 2$)$ was not of statistical significance, roughly two thirds of subjects responded to RT treatment, evidenced by lower glucose and insulin in response to the OGTT. Although not statistically significant, our findings may provide practical aid for selected individuals opting to use such a dietary agent for glucose disposal after carbohydrate-rich feedings. Moreover, we believe that our findings for a response in non-diabetic individuals, with normal fasting glucose and normal glucose tolerance, provide justification for future studies inclusive of larger sample sizes of individuals with impaired glucose tolerance (ie, diabetic, pre-diabetic).

As has been noted for many dietary supplements, ${ }^{24-26} \mathrm{a}$ relatively high degree of subject variability in response to treatment was observed which, when coupled with our relatively small sample size, impaired our chance of detecting statistically significant findings. As this was a pilot study designed to determine if the RT might have an application in larger trials involving diabetic and pre-diabetic patients, our small sample size is justified, and we believe that our objective was met. That is, our results merit attention in future, larger scale trials, in particular those involving individuals with pre-diabetes or untreated diabetes. 
Table 3 Heart rate (HR), systolic blood pressure (SBP), diastolic blood pressure (DBP), and rate pressure product (RPP) before and after ingestion of placebo and Russian tarragon

\begin{tabular}{|c|c|c|c|c|c|c|c|c|}
\hline Time & $\begin{array}{l}\text { HR (bpm) } \\
\text { Placebo }\end{array}$ & $\begin{array}{l}\text { *HR (bpm) } \\
\text { Russian } \\
\text { tarragon }\end{array}$ & $\begin{array}{l}\text { SBP }(\mathrm{mmHg}) \\
\text { Placebo }\end{array}$ & $\begin{array}{l}* \text { SBP }(\mathrm{mmHg}) \\
\text { Russian } \\
\text { tarragon }\end{array}$ & $\begin{array}{l}\text { DBP }(\mathrm{mmHg}) \\
\text { Placebo }\end{array}$ & $\begin{array}{l}\text { *DBP }(\mathrm{mmHg}) \\
\text { Russian } \\
\text { tarragon }\end{array}$ & $\begin{array}{l}\text { RPP } \\
\text { Placebo }\end{array}$ & $\begin{array}{l}* \text { RPP } \\
\text { Russian } \\
\text { tarragon }\end{array}$ \\
\hline Pre & $61 \pm 2$ & $60 \pm 2$ & $112 \pm 2$ & $109 \pm 3$ & $66 \pm 2$ & $62 \pm 3$ & $6870 \pm 239$ & $6571 \pm 249$ \\
\hline $15 \mathrm{~min}$ & $65 \pm 2$ & $61 \pm 2$ & $114 \pm 3$ & $112 \pm 3$ & $65 \pm 2$ & $61 \pm 2$ & $7398 \pm 315$ & $6846 \pm 264$ \\
\hline $30 \mathrm{~min}$ & $63 \pm 2$ & $63 \pm 2$ & $118 \pm 3$ & $112 \pm 3$ & $64 \pm 3$ & $59 \pm 2$ & $7478 \pm 230$ & $7019 \pm 233$ \\
\hline $45 \mathrm{~min}$ & $63 \pm 2$ & $6 I \pm 2$ & $117 \pm 2$ & $113 \pm 3$ & $63 \pm 3$ & $60 \pm 2$ & $7342 \pm 213$ & $6896 \pm 323$ \\
\hline $60 \mathrm{~min}$ & $64 \pm 2$ & $61 \pm 1$ & $116 \pm 2$ & $\| I I \pm 4$ & $64 \pm 3$ & $62 \pm 3$ & $7378 \pm 198$ & $6743 \pm 247$ \\
\hline $75 \mathrm{~min}$ & $63 \pm 2$ & $6 I \pm 2$ & $116 \pm 3$ & $109 \pm 3$ & $67 \pm 2$ & $60 \pm 2$ & $7290 \pm 194$ & $6601 \pm 224$ \\
\hline
\end{tabular}

Notes: Data are mean \pm SEM.

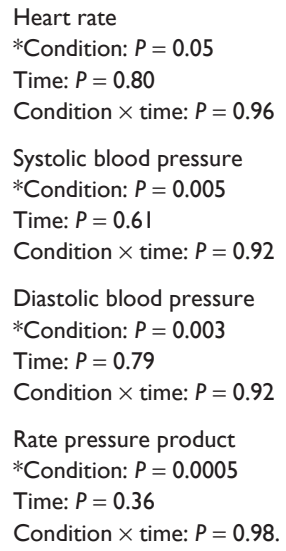

In terms of responders and non-responders to treatment, related to the latter, such individuals may not need additional support in the form of dietary supplementation, as they already possess optimal functioning (ie, have excellent insulin sensitivity and GLUT-4 protein content and activity). Considering this scenario, the addition of a glucose disposal agent such as RT may be unnecessary, as evidenced by no difference in response to treatment compared with a placebo.

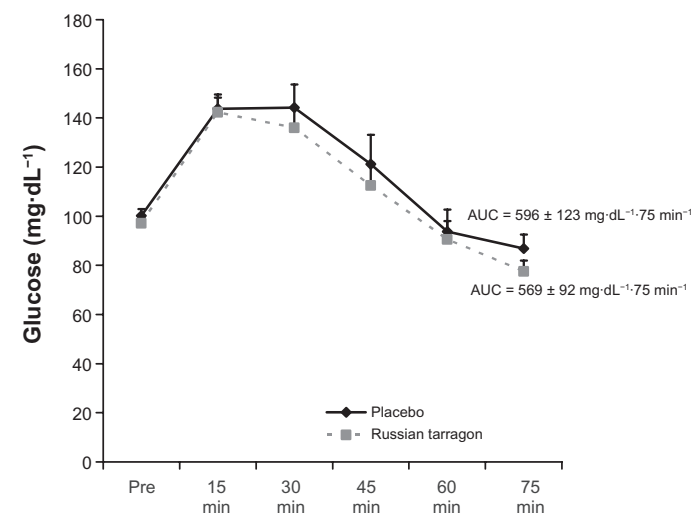

Figure I Serum glucose before and after ingestion of placebo and Russian tarragon. Notes: Data are mean \pm SEM.

Condition: $P=0.19$

Time: $P<0.000 \mathrm{I}$; $15 \mathrm{~min}$ and $30 \mathrm{~min}>$ pre $(P<0.05)$

Condition $\times$ time: $P=0.99$

AUC: $P=0.54$ (4.5\% reduction in AUC compared to placebo).
While this may certainly be true for young, healthy, non-diabetic individuals, older individuals and/or those with impaired glucose tolerance and insulin resistance may respond well to treatment with dietary supplements targeting glucose disposal. Including such individuals as test subjects is a logical next step in future studies investigating the effects of RT on glucose disposal following an OGTT. If promising results are obtained, RT may be considered as an adjunct

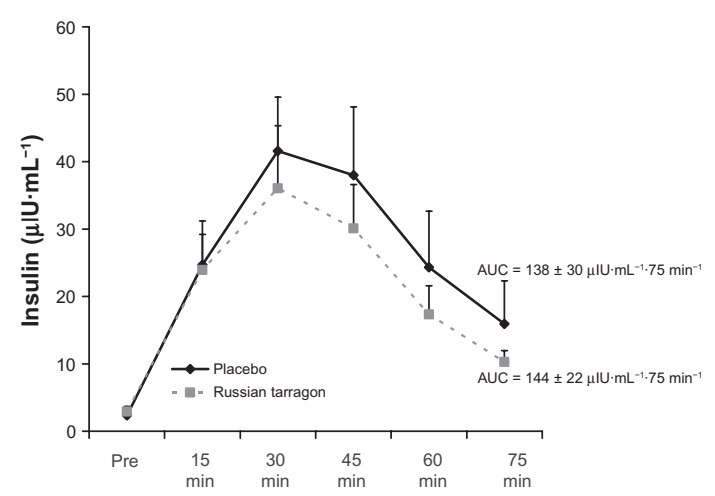

Figure 2 Serum insulin before and following ingestion of placebo and Russian tarragon.

Notes: Data are mean \pm SEM.

Condition: $P=0.24$

Time: $P<0.000$; $15 \mathrm{~min}, 30 \mathrm{~min}$, and $45 \mathrm{~min}>$ pre $(P<0.05)$

Condition $\times$ time: $P=0.98$

AUC: $P=0.53$ ( $17.4 \%$ reduction in AUC compared with placebo). 
therapy for those with impaired glucose tolerance and insulin resistance.

As stated earlier in this paper, an ethanol extract of RT has been developed and reported to exhibit anti-hyperglycemic activity, ${ }^{10}$ which appears to be enhanced when using a bioenhancer/solubilizer. ${ }^{11}$ Other ingredients and species not specifically related to RT have been used for their anti-diabetic effects (eg, Artemisia santonicum); ${ }^{27}$ others are reviewed elsewhere. ${ }^{7,8}$ While it is difficult to make direct comparisons between our findings and those of other ingredients and species, it should be understood that these exist and may provide glucose regulatory benefits similar to RT (Artemisia dracunculus L.). Further work would be needed to provide such comparative data.

Finally, although not a main outcome measure in the present study, hemodynamic variables were all noted to be lower in the RT condition compared with the placebo (Table 3). Although this may be an interesting observation that deserves further attention in a future trial involving a larger number of subjects, and although no condition $\times$ time interaction effects were noted, it appears that the lower pre-ingestion values for these variables contributed most to the condition main effect (as can be seen in Table 3). Alternatively, it is possible that the slight reduction in blood glucose may be associated with a lower production in reactive oxygen species, ${ }^{28}$ which may be related to an improvement in vascular tone, ${ }^{29}$ and hence a lower blood pressure response to the OGTT. Further investigation of the hemodynamic effects after an OGTT with and without RT may provide further clarification.

\section{Conclusion}

Data from the present study indicate that acute ingestion of RT results in a slight, albeit non-statistically significant, lowering of blood glucose in response to an oral dextrose load. The insulin response to the OGTT was also slightly less with RT, but failed to reach statistical significance. These findings are specific to a small sample of young, healthy, non-diabetic men. Additional study is needed using a larger sample of subjects, in particular those with impaired glucose tolerance.

\section{Acknowledgments}

Funding for this work was provided by The University of Memphis and PhytoLab GmbH \& Co. KG. The authors thank Bernd Walbroel and Bjoern Feistel of Finzelberg GmbH \& Co. KG for their support with extract supply.

\section{Author contributions}

RJB was responsible for the study design, overseeing data collection, biochemical work, statistical analysis, and preparation of the manuscript. REC coordinated the study and was responsible for data collection. IP assisted with the study design and manuscript preparation. All authors read and approved the final manuscript.

\section{Disclosure}

RJB has received research funding or acted as consultant to several nutraceutical and dietary supplement companies. IP is an employee of PhytoLab GmbH \& Co. KG. REC declares no competing interests.

\section{References}

1. Centers for Disease Control and Prevention. National diabetes fact sheet: General information and national estimates on diabetes in the United States, 2007. Atlanta, GA: US Department of Health and Human Services; 2008. http://www.cdc.gov/diabetes/pubs/pdf/ndfs_2007.pdf. Accessed January 6, 2011.

2. Lo MC, Lansang MC. Recent and emerging therapeutic medications in type 2 diabetes mellitus: Incretin-based, pramlintide, colesevelam, SGLT2 inhibitors, tagatose, succinobucol. Am J Ther. 2010 [Epub ahead of print].

3. Praet SF, van Loon LJ. Optimizing the therapeutic benefits of exercise in type 2 diabetes. J Appl Physiol. 2007;103(4):1113-1120.

4. Nield L, Moore HJ, Hooper L, et al. Dietary advice for treatment of type 2 diabetes mellitus in adults. Cochrane Database Syst Rev. 2007; (3):CD004097.

5. Fonseca VA, Zinman B, Nauck MA, Goldfine AB, Plutzky J. Confronting the type 2 diabetes epidemic: the emerging role of incretin-based therapies. Am J Med. 2010;123(7):S2-S10.

6. Bartlett HE, Eperjesi F. Nutritional supplementation for type 2 diabetes: a systematic review. Ophthalmic Physiol Opt. 2008;28(6):503-523.

7. Cefalu WT, Ye J, Zuberi A, et al. Botanicals and the metabolic syndrome. Am J Clin Nutr. 2008;87(2):481S-487S.

8. Cefalu WT, Ye J, Wang ZQ. Efficacy of dietary supplementation with botanicals on carbohydrate metabolism in humans. Endocr Metab Immune Disord Drug Targets. 2008;8(2):78-81.

9. Zuberi AR. Strategies for assessment of botanical action on metabolic syndrome in the mouse and evidence for a genotype-specific effect of russian tarragon in the regulation of insulin sensitivity. Metabolism. 2008;57(7 Suppl 1):S10-S15.

10. Ribnicky DM, Poulev A, Watford M, Cefalu WT, Raskin I. Antihyperglycemic activity of Tarralin, an ethanolic extract of Artemisia dracunculus L. Phytomedicine. 2006;13(8):550-557.

11. Ribnicky DM, Kuhn P, Poulev A, et al. Improved absorption and bioactivity of active compounds from an anti-diabetic extract of Artemisia dracunculus L. Int J Pharm. 2009;370(1-2):87-92.

12. Wang ZQ, Ribnicky D, Zhang XH, Raskin I, Yu Y, Cefalu WT. Bioactives of Artemisia dracunculus $\mathrm{L}$ enhance cellular insulin signaling in primary human skeletal muscle culture. Metabolism. 2008; 57(7 Suppl 1):S58-S64.

13. Govorko D, Logendra S, Wang Y, et al. Polyphenolic compounds from Artemisia dracunculus L. inhibit PEPCK gene expression and gluconeogenesis in an H4IIE hepatoma cell line. Am J Physiol Endocrinol Metab. 2007;293(6):E1503-E1510.

14. Walbroel B, Feistel B, Pischel I. Russian tarragon (Artemisia dracunculus L.) extracts and their antidiabetic potentials. 13th International Congress, PhytoPharm 2009, Bonn, Germany. 
15. Pischel I, Walbroel B, Fiestel B, inventors. Water-soluble extracts of Artemisia dracunculus (Tarragon) for improvement of glucose metabolism. Patent WO/2009/147228. 10.12.2009, 2009.

16. Maki KC, McKenney JM, Farmer MV, Reeves MS, Dicklin MR. Indices of insulin sensitivity and secretion from a standard liquid meal test in subjects with type 2 diabetes, impaired or normal fasting glucose. Nutr J. 2009;8:22.

17. Bloomer RJ, Kabir MM, Marshall KE, Canale RE, Farney TM. Postprandial oxidative stress in response to dextrose and lipid meals of differing size. Lipids Health Dis. 2010;9(1):79.

18. Monnier L, Mas E, Ginet C, et al. Activation of oxidative stress by acute glucose fluctuations compared with sustained chronic hyperglycemia in patients with type 2 diabetes. JAMA. 2006;295(14): 1681-1687.

19. Ceriello A, Taboga C, Tonutti L, et al. Evidence for an independent and cumulative effect of postprandial hypertriglyceridemia and hyperglycemia on endothelial dysfunction and oxidative stress generation: Effects of short- and long-term simvastatin treatment. Circulation 2002;106(10):1211-1218.

20. Lee IK, Kim HS, Bae JH. Endothelial dysfunction: its relationship with acute hyperglycaemia and hyperlipidemia. Int J Clin Pract Suppl. 2002; (129):59-64.

21. King DS, Baldus PJ, Sharp RL, Kesl LD, Feltmeyer TL, Riddle MS. Time course for exercise-induced alterations in insulin action and glucose tolerance in middle-aged people. J Appl Physiol. 1995;78(1): $17-22$.
22. Brestoff JR, Clippinger B, Spinella T, von Duvillard SP, Nindl BC, Arciero PJ. An acute bout of endurance exercise but not sprint interval exercise enhances insulin sensitivity. Appl Physiol Nutr Metab. 2009;34(1):25-32.

23. Pruessner JC, Kirschbaum C, Meinlschmid G, Hellhammer DH. Two formulas for computation of the area under the curve represent measures of total hormone concentration versus time-dependent change. Psychoneuroendocrinology. 2003;28(7):916-931.

24. Bloomer RJ, Smith WA, Fisher-Wellman KH. Glycine propionylL-carnitine increases plasma nitrate/nitrite in resistance trained men. J Int Soc Sports Nutr. 2007;4:22.

25. Ganio MS, Klau JF, Casa DJ, Armstrong LE, Maresh CM. Effect of caffeine on sport-specific endurance performance: a systematic review. $J$ Strength Cond Res. 2009;23(1):315-324.

26. Hadjicharalambous M, Kilduff LP, Pitsiladis YP. Brain serotonin and dopamine modulators, perceptual responses and endurance performance during exercise in the heat following creatine supplementation. J Int Soc Sports Nutr. 2008;5:14.

27. Korkmaz H, Gürdal A. Effect of Artemisia santonicum L. on blood glucose in normal and alloxan-induced diabetic rabbits. Phytother Res 2002;16(7):675-676.

28. Mohanty P, Hamouda W, Garg R, Aljada A, Ghanim H, Dandona P. Glucose challenge stimulates reactive oxygen species (ROS) generation by leucocytes. J Clin Endocrinol Metab. 2000;85(8):2970-2973.

29. Bauer V, Sotníková R. Nitric oxide - the endothelium-derived relaxing factor and its role in endothelial functions. Gen Physiol Biophys. 2010; 29(4):319-340.
Nutrition and Dietary Supplements

\section{Publish your work in this journal}

Nutrition and Dietary Supplements is an international, peer-reviewed, open access journal focusing on research into nutritional requirements in health and disease, impact on metabolism and the identification and optimal use of dietary strategies and supplements necessary for normal growth and development. The journal welcomes papers covering origi-

\section{Dovepress}

nal research, basic science, clinical \& epidemiological studies, reviews and evaluations, guidelines, expert opinion and commentary, case reports and extended reports. The manuscript management system is completely online and includes a very quick and fair peer-review system, which is all easy to use. 